\title{
Review Article \\ Optical Forces in Silicon Nanophotonics and Optomechanical Systems: Science and Applications
}

\author{
Lip Ket Chin $\mathbb{D}^{1,2}$ Yuzhi Shi $\mathbb{D}^{2}$ and Ai-Qun Liu $\mathbb{D}^{2}$ \\ ${ }^{1}$ Center for Systems Biology, Massachusetts General Hospital, Boston, MA, USA \\ ${ }^{2}$ School of Electrical and Electronic Engineering, Nanyang Technological University, Singapore \\ Correspondence should be addressed to Lip Ket Chin; lkchin@mgh.harvard.edu and Ai-Qun Liu; eaqliu@ntu.edu.sg
}

Received 15 July 2020; Accepted 22 September 2020; Published 26 October 2020

Copyright (c) 2020 Lip Ket Chin et al. Exclusive Licensee Beijing Institute of Aerospace Control Devices. Distributed under a Creative Commons Attribution License (CC BY 4.0).

\begin{abstract}
Light-matter interactions have been explored for more than 40 years to achieve physical modulation of nanostructures or the manipulation of nanoparticle/biomolecule. Silicon photonics is a mature technology with standard fabrication techniques to fabricate micro- and nano-sized structures with a wide range of material properties (silicon oxides, silicon nitrides, $p$ - and $n$ doping, etc.), high dielectric properties, high integration compatibility, and high biocompatibilities. Owing to these superior characteristics, silicon photonics is a promising approach to demonstrate optical force-based integrated devices and systems for practical applications. In this paper, we provide an overview of optical force in silicon nanophotonic and optomechanical systems and their latest technological development. First, we discuss various types of optical forces in light-matter interactions from particles or nanostructures. We then present particle manipulation in silicon nanophotonics and highlight its applications in biological and biomedical fields. Next, we discuss nanostructure mechanical modulation in silicon optomechanical devices, presenting their applications in photonic network, quantum physics, phonon manipulation, physical sensors, etc. Finally, we discuss the future perspective of optical force-based integrated silicon photonics.
\end{abstract}

\section{Introduction}

Over 40 years of discoveries and developments, optical forces have been studied intensively and employed either for the physical modulation of nanostructures or the manipulation of nanoparticle/biomolecule, even marching into the atom realm by the assist of the optical cooling technique $[1,2]$. Optical forces thus find themselves huge potentials in physical, biomedical, and chemical sciences.

Conventionally, optical force is widely demonstrated in far-field approaches using the fundamental optical mode (normally Gaussian) for micro-sized particle [3-5] and cell manipulation [6, 7]. However, such approaches are hindered by diffraction limit of light and complexities in the configuration of the large area of uniform optical fields. As a result, farfield approaches might not be suitable for the manipulation of nano-sized particles (such as deoxyribonucleic acids, virus particles, and exosomes) or modulation of nanostructures. Plasmonic approaches, on the other hand, take the advantage of highly localized optical field generated by surface plasmon resonances to trap nanoparticles rapidly $[8,9]$. Unfortu- nately, the huge heat generated by the localized optical field and induced vector flow are two unavoidable severe problems associated with plasmonic approaches. This heating effect could damage the structure of biological nanoparticles, limiting the practical applications in biological and biomedical fields.

Silicon photonics is a mature technology with standard fabrication techniques to fabricate micro- and nano-sized structures, which include mechanical components such as actuators, sensors, and optical components such as lens, mirrors, and lasers [10-12]. Its wide range of material properties (silicon oxides, silicon nitrides, $p$ - and $n$-doping, etc.), high dielectric properties, high integration compatibility with complementary metal-oxide semiconductor platform and other materials, capability of mass production, and high biocompatibilities show that silicon photonics is a promising approach to demonstrate optical force-based integrated devices and systems for practical applications.

In this paper, we provide an overview of optical force in silicon nanophotonic and optomechanical systems and their latest technological development. We also present their 
applications in biosensing, biomedicine, quantum physics, etc., and discuss future perspective of optical force-based integrated silicon photonics.

\section{Theoretical Principles of Optical Force}

The general approaches to harness optical forces in silicon nanophotonics are illustrated in Figure 1. The most intuitive way is using an optical waveguide structure or a ring resonator to confine light as shown in Figure 1(a). In this case, the silicon micro-/nano-structure stores photons and interacts with the particles. Light in the waveguide or ring resonator exchanges momentum with the particle by the evanescence wave, which attracts particle to the surface of the waveguide. The particle can be pushed by the radiation pressure or trapped inside potential wells depending on the configurations of the waveguide. The optical gradient force, which serves as the trapping force for a Rayleigh particle (radius $<<$ wavelength) being placed within the evanescence wave above the nanostructure, can be expressed as $[13,14]$.

$$
F_{\text {grad }}=\frac{2 \pi n_{2} r^{3}}{c}\left(\frac{m^{2}-1}{m^{2}+2}\right) \nabla I,
$$

where $m=n_{1} / n_{2}, r$ is the radius of the particle, $n_{1}$ and $n_{2}$ are the refractive index of the particle and the medium, respectively, $c$ is the speed of light in vacuum, and $I$ is the light intensity. The optical gradient force can be increased by minimizing the size of the optical hotspot, i.e., increasing the gradient of the intensity change $[15,16]$. The optical scattering force and absorption force on a dielectric particle predicted by the Rayleigh theory can be expressed as $[13,17]$.

$$
F_{s c a}=\frac{128 \pi^{5} n_{2} r^{6} I}{3 c \lambda^{4}}\left(\frac{m^{2}-1}{m^{2}+2}\right)^{2}
$$

and

$$
F_{a b s}=\frac{8 \pi^{2} n_{2} r^{3} I}{c \lambda} \operatorname{Im}\left(\frac{m^{2}-1}{m^{2}+2}\right),
$$

where $\lambda$ is the wavelength of light. The combination of the optical scattering and absorption forces is known as the optical extinction force, which acts to push or pull the particle. The efficiency of the transport of the nanoparticles can be improved by increasing the light intensity.

Optical forces can be enhanced by using resonant cavity such as the ring resonator and photonic crystal, whereby light is confined in subwavelength $(<1 / 2 \lambda)$ structures with strong photon resonance via the whispering gallery mode [18], Fabry-Pérot cavities [19], guided mode [20], Bloch mode [21], etc. The intensity enhancement factors could be few hundreds higher than the input laser intensity. Therefore, the intensity gradient $\Delta T$ is hugely enhanced, as well as the associated optical gradient force, which can be used for the manipulation of nano-sized bioparticles, such as bacteria [22], virus [23], and DNA [20].
A distinctive type of optical lateral force exists on a chiral particle that is placed above a substrate (metallic or dielectric), emerging from the coupling between the chiral particle and the reflected light from the substrate surface [24]. The chiral particle is pushed sideways (perpendicular to the light propagation and in-plane with the substrate), and the direction depends on the chirality. This optical lateral force originates from the lateral radiation pressure and the optical spin density force, coupling the chirality of the particle to the lateral linear momentum and spin angular momentum. The lateral force is larger when the particle is nearer to the substrate due to the asymmetrical coupling between them. The optical lateral force can also act on any particle (non-chiral, symmetric) near a surface by using an incident circularly polarized light through spin-orbit coupling (Figure 1(b)) [25]. With circularly polarized light, an asymmetric and unidirectional scattering is achieved, creating an equal and opposite mechanical momentum. Particles can also foresee rotating in an optical vortex emitters using angular gratings to extract light confined in whispering gallery modes [26]. The rotation relies on the transfer of orbital angular momentum from light to the particles [27].

Besides light-particle interaction, optical forces also act on suspended nanowaveguides through interactions between them (Figure 1(c)), causing the suspended nanowaveguides to be pulled by the substrate [28]. In this case, the optical force origins from the coupling of light between two silicon nanostructures. The optical forces acted on the two suspended waveguides can be attractive or repulsive forces between each other when they are closely coupled $[28,29]$. The optical force emerges when one waveguide is placed in the light field of another, which can be simulated from the integration of stress tensors [30-32]. The Maxwell stress tensor is expressed as [33].

$$
T_{i j}=\varepsilon_{0} E_{i} E_{j}+\mu_{0} H_{i} H_{j}-\frac{1}{2}\left(\varepsilon_{0} E^{2}+\mu_{0} H^{2}\right) \delta_{i j}
$$

where $\varepsilon_{0}$ and $\mu_{0}$ are the electric and magnetic constants, respectively; $E_{i}$ and $E_{j}$ are components of the electric field $E$ ; $H_{i}$ and $H_{j}$ are the components of magnetic induction $H$; and $\delta_{i j}$ is the Kronecker delta.

The Minkowski stress tensor is express as

$$
T_{i j}=E_{i} D_{j}+H_{i} B_{j}-\frac{1}{2}(\mathbf{E D}+\mathbf{H B}) \delta_{i j}
$$

where $\mathbf{D}=\varepsilon \varepsilon_{0} \mathbf{E}$ and $\mathbf{B}=\mu \mu_{0} \mathbf{H} . \varepsilon$ and $\mu$ are the permittivity and permeability of the medium, respectively.

The optical force then can be expressed as [34].

$$
\langle F\rangle=\oint_{s}\langle T\rangle d S
$$

where the integration is performed over a closed surface near the object, and \langle\rangle represents the time average operation. In the vacuum environment, the results calculated from Maxwell and Minkowski stress tensors are identical. However, the Minkowski stress tensor is more widely used in liquids 


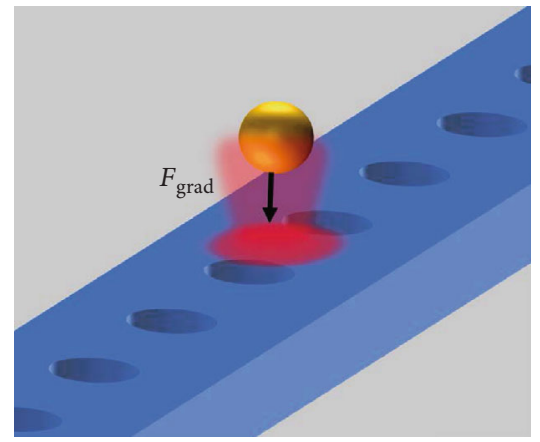

(a)

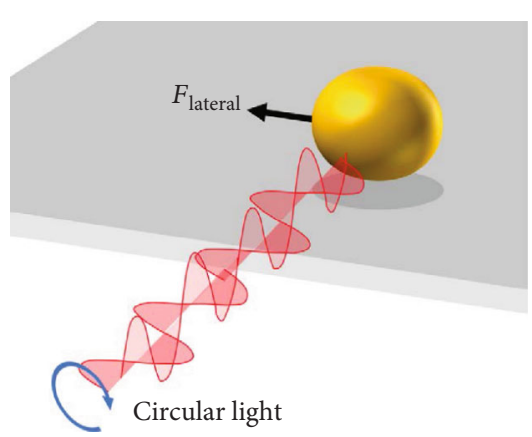

(b)

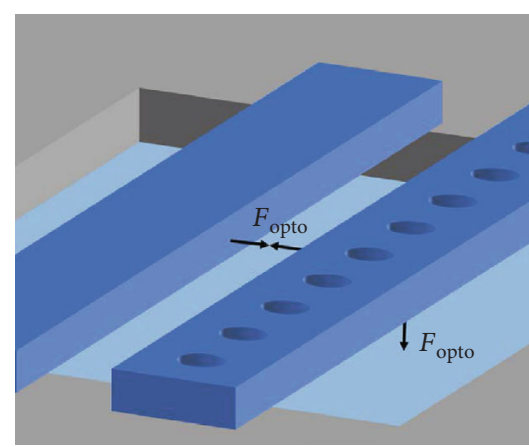

(c)

FIGURE 1: Optical forces in light-particle and light-nanostructure interactions. (a) Optical gradient force acted on a nanoparticle in vicinity to the resonant nanowaveguide. (b) Optical lateral force acted on the particle in vicinity to a surface using circular polarized light. (c) Optical force acted on resonant nanostructures in optomechanical devices, leading to mechanical vibrational resonance.

TABLE 1: Summary of the optical forces in silicon nanophotonics and optomechanics.

\begin{tabular}{|c|c|c|c|c|c|}
\hline Mechanism & & Material & $\begin{array}{l}\text { Optical } \\
\text { force/stiffness }\end{array}$ & $\begin{array}{l}\text { Trapping quantity } \\
(>50)\end{array}$ & Ref \\
\hline \multirow{6}{*}{ Light particle } & Waveguide & Polystyrene, $200 \mathrm{~nm}$ & $\sim 55 \mathrm{pN} / \mathrm{W}$ & Yes & [39] \\
\hline & Slot waveguide & $\begin{array}{l}\text { DNA and polystyrene, } \\
100 \mathrm{~nm}\end{array}$ & $\sim 25 \mathrm{pN} / \mathrm{W}$ & No & [20] \\
\hline & Ring resonator & Polystyrene, $1.1 \mu \mathrm{m}$ & $\sim 0.15 \mathrm{nN} / \mathrm{W}$ & No & [18] \\
\hline & Photonic crystal (defect mode) & Polystyrene, $100 \mathrm{~nm}$ & $\sim 700 \mathrm{pN} / \mathrm{W}$ & Yes & [40] \\
\hline & Photonic crystal (guided mode) & Polystyrene, $520 \mathrm{~nm}$ & $\sim 5 \mathrm{nN} / \mathrm{W}$ & Yes & [41] \\
\hline & Silicon substrate (lateral force) & Gold, $40 \mathrm{~nm}$ & $\underset{2}{\sim 0.4 \mathrm{pN} /\left(\mathrm{mW} \mu \mathrm{m}^{-}\right.}$ & - & [24] \\
\hline \multirow{4}{*}{$\begin{array}{l}\text { Light } \\
\text { nanostructure }\end{array}$} & Nanowaveguide and substrate & Silicon, $500 \mathrm{~nm}$ (width) & $\sim 0.5 \mathrm{pN} /(\mu \mathrm{m} \mathrm{mW})$ & - & {$[28]$} \\
\hline & Ring resonator and substrate & Silicon, $450 \mathrm{~nm}$ (width) & $\sim 50 \mathrm{nN} /(\mu \mathrm{m} \mathrm{mW})$ & - & [42] \\
\hline & Parallel ring resonators & $\begin{array}{l}\text { Silicon nitride, } 2.5 \mu \mathrm{m} \\
\text { (width) }\end{array}$ & $\sim 20 \mathrm{nN} /(\mu \mathrm{m} \mathrm{mW})$ & - & [43] \\
\hline & $\begin{array}{l}\text { Dual nanowaveguide (Casimir } \\
\text { force) }\end{array}$ & Silicon, $500 \mathrm{~nm}$ (width) & $\sim 1 \mathrm{pN} /(\mu \mathrm{m} \mathrm{mW})$ & - & [38] \\
\hline
\end{tabular}

[34]. Similarly, the optical forces between two nanostructures can be enhanced by resonant cavities.

In addition to optical gradient force, there also exists optical force mediated by virtual photons, which is known as the Casimir force [35-37], which has to be considered especially in coupled nanostructures [38]. Although Casimir force is reduced at least by a factor of three in silicon as compared to metallic nanostructures, it is significant when the gap between two nanostructures is less than $100 \mathrm{~nm}$, in the order of $\mathrm{pN} / \mu \mathrm{m}$. Table 1 summarized optical forces in silicon nanophotonics and optomechanical systems.

\section{Optical Force in Light-Particle Interactions}

3.1. Silicon Nanophotonics for Particle Manipulation. Silicon nanowaveguide is one of the structural designs widely used to trap near-infrared light (e.g., 1,550 nm) and generate optical force through evanescence fields to manipulate nanoparticles [45-48]. In addition to the widely used fundamental transverse electric $\mathrm{TE}_{0}$ mode light in the nanowaveguide, high-order TE and transverse magnetic (TM) modes can also be employed for versatile particle manipulation. Pin et al. designed a silicon waveguide with a cross-sectional dimension of $510 \mathrm{~nm} \times 248 \mathrm{~nm}$, maximizing the difference in the effective refractive index between three guided modes, $\mathrm{TE}_{0}$, $\mathrm{TM}_{0}$, and $\mathrm{TE}_{1}[44]$. Three different trapping regimes resulting from the copropagation of different guided modes were achieved by using different light coupling conditions (Figure 2(a)), showing the stable trapping of large ensemble of polystyrene microbeads and bacteria. With the increasing demand on the trapping of nanoparticles down to the sub$100 \mathrm{~nm}$, Yang et al. designed a slot waveguide with a slot less than $100 \mathrm{~nm}$ to condense light to a high intensity level $\left(10^{4} \mathrm{~mW} / \mu \mathrm{m}^{2}\right)$ [49]. The slot waveguide could exert a piconewton optical gradient force on the $70 \mathrm{~nm}$ polystyrene nanoparticle for effective trapping with a trapping stiffness of $\sim 0.2 \mathrm{pN} /(\mathrm{nm} \cdot \mathrm{W})$. In addition, a slot nanobeam cavity was proposed to confine light in the deep subwavelength scale by introducing nanocavities in the slot waveguide, which, theoretically, can trap a $2 \mathrm{~nm}$ nanoparticle in the cavity with an ultrahigh trapping stiffness of $\sim 0.4$ $\mathrm{pN} /(\mathrm{nm} \cdot \mathrm{mW})[50]$. 


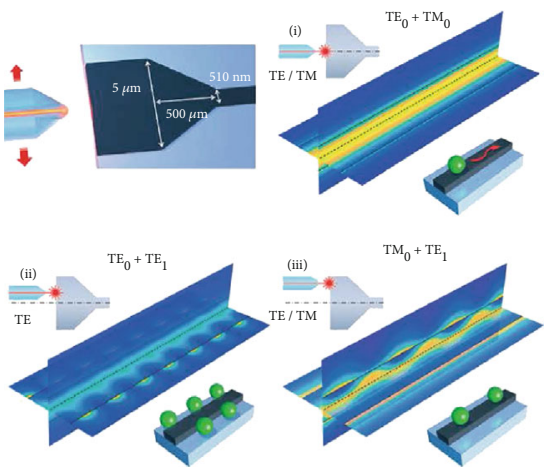

(a)
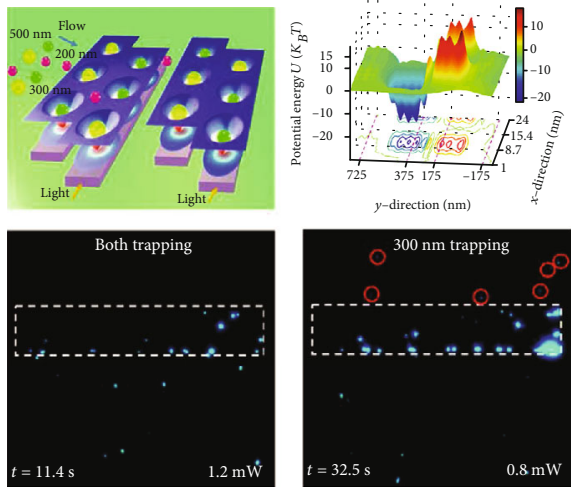

(b)
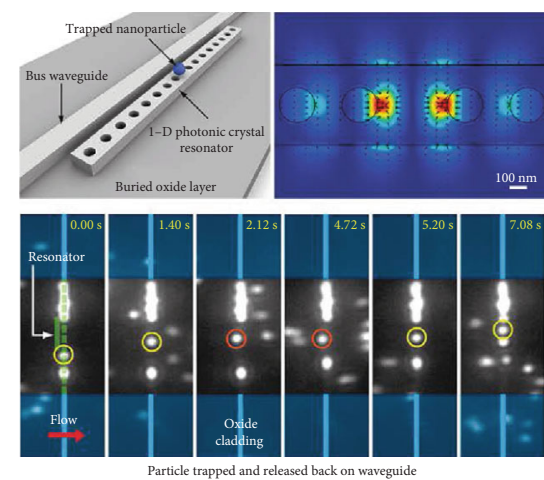

(c)

FIGURE 2: Silicon nanophotonics for particle manipulation. (a) Three different trapping schemes in nanowaveguide using different coupling conditions, reprinted with permission from ref. [44]. (b) Massive trapping and sorting of nanoparticles in a coupled optical potential well array, reprinted with permission from ref. [39]. (c) Particle trapping in a photonic crystal waveguide, reprinted with permission from ref. [40].

In addition to particle trapping, Lin et al. designed a channel waveguide and a slot waveguide, forming an optical splitter to sort nanoparticles from microparticles [51]. The waveguides are separated by $200 \mathrm{~nm}$, generating two potential wells for nanoparticles, but a broad potential well for the larger particles. As a result, nanoparticles were transferred to the slot waveguide with a structural perturbation consisting of a stuck bead and microparticles that followed the channel waveguide, which was associated with a deeper potential well. Multilevel sorting of different sized nanoparticles was also proposed on a multistep waveguide splitter [52] or an array of nanowaveguide pairs (Figure 2(b)) [39].

Compared to a waveguide trapping configuration, light oscillates in a high-quality factor ring resonator and results in enhanced optical field and optical forces [53]. By tuning the resonance to the whispering gallery mode, particles were propelled around the ring at hundreds of micrometers per second, producing periodic revolutions at a few hertz [18]. As the crosssectional area of the microring is normally the same as the nanowaveguide, light enhancement of the ring can be regarded as the enhancement of the nanowaveguide multiplied by the ring enhancement factor. Slot waveguide can also be used to design ring resonators [54]. The slot ring resonator serves as an alternative method to trap and detect small particle quantity in the clusters in ultra-low concentration $(\sim \mathrm{fM})$ with several orders of magnitude better sensitivity than single ring resonators.

An optical ring resonator switch consists of a bus waveguide being coupled to a ring resonator that was achieved by tuning the wavelength of input light [55]. When the input light is in resonant with the ring resonator, particles will be transport from the bus waveguide to the ring resonator. Alternatively, the resonance mode of the ring resonator can also be tuned thermally through the integration of a microheater. Various functions can be realized, including particle sorting, storage, and mixing [56]. In addition, Xu et al. proposed a cascaded ring-assisted Mach-Zehnder interferometer for multilevel nanoparticle sorting [57]. By heating the ring resonator locally, the optical power ratio between parallel waveguides in the Mach-Zehnder interferometer is tuned, leading to the change of the induced optical potential well and resulting in different particle transferring thresholds for different power ratios.

Photonic crystal structures inscribed in a slab waveguide associates total internal reflection with the photonic bandgap effect to achieve enhanced photon confinement, while preserving a great potential for integration in complex photonic architectures [58]. An enhanced light confinement in photonic crystals creates large field gradients of the electromagnetic field intensity. These strong field gradients coupled with the resonant amplification of the optical field within the resonator enable the stable trapping of particles ranging in size from 50 to $500 \mathrm{~nm}$ (Figure 2(c)) [40]. Optimizing structural designs of the photonic crystal waveguide cavity further enhances the resonance and optical trap. For instance, photonic crystal waveguide with a waist structure lowers the threshold power for stable trapping [59]; the slotted photonic crystal cavity enables the trapping of $10 \mathrm{~nm}$ nanoparticles with enhanced trapping force in the order of $\mathrm{nN} / \mathrm{mW}$ [60]; and a bow-tie-shaped photonic crystal nanobeam cavity can theoretically trap nanoparticles as small as $3 \mathrm{~nm}$ with a maximum trapping force of $10^{2} \mathrm{nN} / \mathrm{mW}$ [61]. Two-dimensional photonic crystals were used for patterned optical trapping of nanoparticles with a laser being loosely focused on the surface of the photonic crystals, generating a patterned optical diffraction field [62]. Templated, self-assembly of nanoparticles $(520 \mathrm{~nm}$ particles and $200 \mathrm{~nm}$ gold particles) was also demonstrated, whereby the resonantly enhanced near field in the photonic crystals creates periodically spaced optical traps $[41,63]$.

3.2. Light-Particle Interactions for Biomedical Applications. Integrated silicon nanophotonic devices with microfluidic technology are useful to trap cells such as red blood cells and yeast cells $[45,64]$ and also characterize single cell noninvasively [65]. Furthermore, trapped cell can be used as a biomagnifier to magnify and image nanostructures a resolution of $100 \mathrm{~nm}$ [66].

To trap smaller single bacterium, a nanowaveguide with the 1D microcavity resonator and a $Q$-factor of 4,000 was designed for biophysical characterization [22]. The trapped bacterium on the microcavity resonator leads to the shift of 
the resonant wavelength, which is correlated to the refractive index of the bacterium. Therefore, by monitoring the spatial and temporal variations of the transmission intensity, the refractive index of a single bacterium can be determined. Similar work using the 2D hollow photonic crystal cavity was also demonstrated to trap [67] and differentiate grampositive and gram-negative bacteria [68]. The photonic crystal cavity (diameter of lattice holes: $250 \mathrm{~nm}$, lattice constant: $420 \mathrm{~nm}$, diameter of defect hole: $700 \mathrm{~nm}$ ) has a resonant wavelength of $1,550 \mathrm{~nm}$ and a $Q$-factor of 4,500 in water. The results showed that gram-negative bacteria exhibit larger transmission increase, which is corresponding to larger resonant wavelength shift and higher refractive index. However, this measurement approach cannot differentiate specific bacteria strains due to the overlapped refractive index variation of the bacteria. Conteduca et al. used the silicon photonic crystal cavity with metal electrodes to trap single bacterium for antimicrobial resistance studies [69]. In addition to optical properties (transmission and resonance shift), the impedance of the surrounding medium is monitored, which is correlated to the metabolic rate in response to antibiotics. Parts of the photonic crystals are heavily doped to confine the electrical current flow within the silicon slab, and the silicon cavity used to trap single bacterium is undoped to allow current flowing through the medium in the trapping region. A detectable difference of $1.2 \mathrm{nA}$ of the current variation was measured between live and dead bacteria.

To manipulate multiple bacteria for high-throughput studies, an array of nanowaveguide pairs was designed by engineering the optical lattice pattern and associated optical force field [71]. The 16 nanowaveguide pairs (350 $\mathrm{nm}$ width, $220 \mathrm{~nm}$ height, $100 \mu \mathrm{m}$ long) are connected via 4-stage lowloss beam splitters. The gap between two waveguides is $200 \mathrm{~nm}$, and the distance between adjacent waveguide pairs is $1 \mu \mathrm{m}$. Bacteria passing through the nanowaveguide array were trapped by optical force and rotated by optical torque, aligning themselves along the nanowaveguides. In situ viability studies based on $20 \%$ ethanol solution treatment were performed via viability fluorescence staining. In addition, the similar platform was used for shape-selective sieving of bacteria (Figure 3(a)) [70]. By optimizing the nanowaveguide physical parameters, laser power, and microfluidic flow velocity, the nanowaveguide pairs can separate spherical Staphylococcus aureus (S. aureus, $\sim 600 \mathrm{~nm}$ diameter) and rod-shaped Escherichia coli (E. coli, $\sim 2 \mu \mathrm{m}$ long, $\sim 500 \mathrm{~nm}$ diameter). When a larger $E$. coli is temporarily trapped in an optical hotspot, it is also under influence of the nearby hotspot. The optical gradient force from nearby hotspot attracts the E. coli, causing it to rotate with a torque and eventually escapes from the unstable optical trap. With a laser power of $1 \mathrm{~mW}$ and a flow velocity of $\sim 6 \mu \mathrm{m} / \mathrm{s}$, more than $95 \%$ of $S$. aureus were trapped in the nanowaveguide array, but only less than $3 \%$ of $E$. coli were trapped. The flow velocity can be further increased for the shaped-based sieving using higher laser power, e.g., $2 \mathrm{~mW}$.

The optical force-based silicon nanophotonics is also capable to manipulate smaller bioparticles and biomolecules such as virus [23] and DNA molecules [20, 72]. Kang et al. used a nanowaveguide resonant cavity to trap a single
H1N1 influenza virus and measure the stoichiometry of antibody binding interactions. A near-field light scattering technique was employed to analyze the change of Brownian fluctuations of the trapped virus particle before and after antibody binding. A stoichiometry result of $26 \pm 4$ antiinfluenza antibodies binding to an $\mathrm{H} 1 \mathrm{~N} 1$ influenza virus was reported (Figure 3(b)), which is consistent with the one reported using fluorescence immunolabeling observed under total internal reflection microscopy [73]. To trap even smaller biomolecules such as DNA, nanowaveguides with smaller dimensions are needed to better confine light in the waveguide, creating a higher optical force. A $60 \mathrm{~nm}$ slot nanowaveguide was successfully used to trap 48 kilobases $\lambda$-DNA molecules with a $250 \mathrm{~mW}$ optical power [20]. Soltani et al. also demonstrated the stretching of DNA molecules by attaching the two ends of $10 \mathrm{kbp}$ DNA molecules with $490 \mathrm{~nm}$ beads and trapping the beads on two separated nanowaveguides [72]. By tuning the phase change via the thermooptic effect, nanometer resolution control of the bead position was achieved. By displacing the distance between the two beads, the DNA molecules was stretched, which is useful for the studies of the structure, chemical bonding, and mechanical properties of DNA molecules.

\section{Optical Force in Light- Nanostructure Interactions}

4.1. Silicon Optomechanical Systems. Optical forces are not only originated from the light-particle interactions and used for nanoparticle manipulation but also exist for the interactions between two nanostructures in silicon optomechanical systems. The first experimental demonstration of optical forces being acted on a suspended nanowaveguide was done by Li et al. in 2008 [28]. They observed a pN optical attraction force on the suspending nanowaveguide $\left(8 \mathrm{pN} \mu \mathrm{m}^{-1} \mathrm{~mW}^{-1}\right.$ when the gap is $50 \mathrm{~nm}$ ), arising from the evanescent coupling of the guided light to the dielectric silicon dioxide substrate. An optical repulsive force was then discovered between two suspending nanowaveguides by tuning the phase from the symmetric to asymmetric modes $[76,77]$. The optical repulsive force is further studied in the case, whereby the optomechanical device is immersed in the fluid media [78]. Based on a slot-waveguide structure, the optical repulsive force increases with increasing the fluid medium's refractive index under the condition of the same slot gap. These studies provide design guidelines for novel optomechanical systems integrated with microfluidic functionalities, which are useful for the lab-on-a-chip application.

Optomechanically coupled cavity resonances, such as ring resonators, can give rise to strong and highly localized optomechanical potential wells [79]. Optical attractive or repulsive forces can be obtained by tuning the pump laser towards the symmetric or asymmetric resonance mode [43]. When the laser wavelength excites the WGM, the optical forces increases extraordinarily since more optical energy under resonance is stored in the cavity. The ring resonator enhances the optical gradient force, allowing nanostructure manipulation at a relatively low light power. When two ring resonators were aligned vertically with a nano-range gap, a 

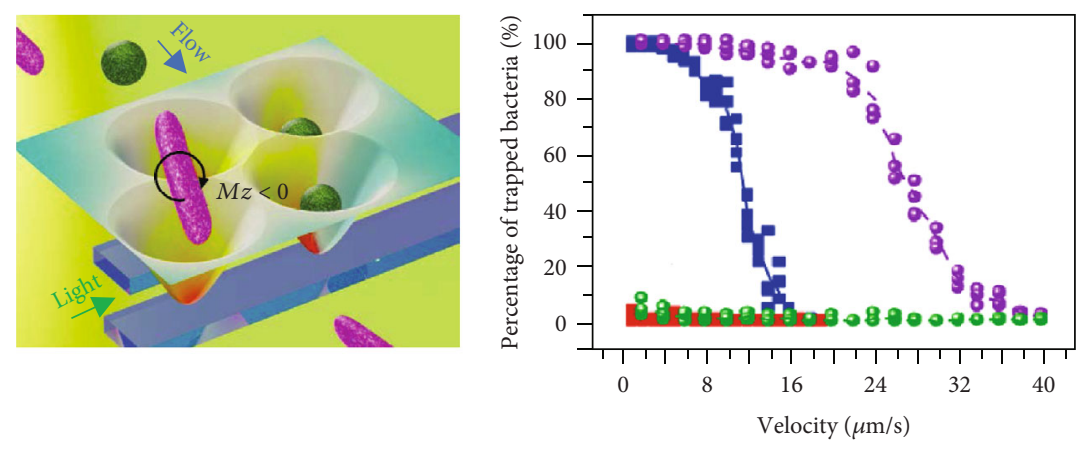

$$
\begin{aligned}
& \text { - S.aureus - S.aureus }(2 \mathrm{~mW}) \\
& \text { - Average - - Average }(2 \mathrm{~mW} \\
& \text { - E.coli - E.coli }(2 \mathrm{~mW}) \\
& \text { Average - - Average }(2 \mathrm{~mW})
\end{aligned}
$$
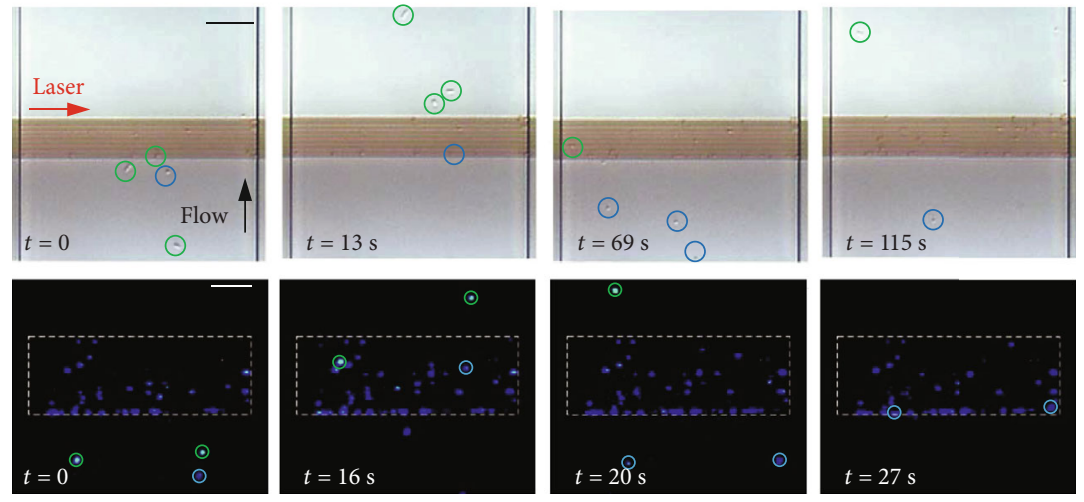

(a)
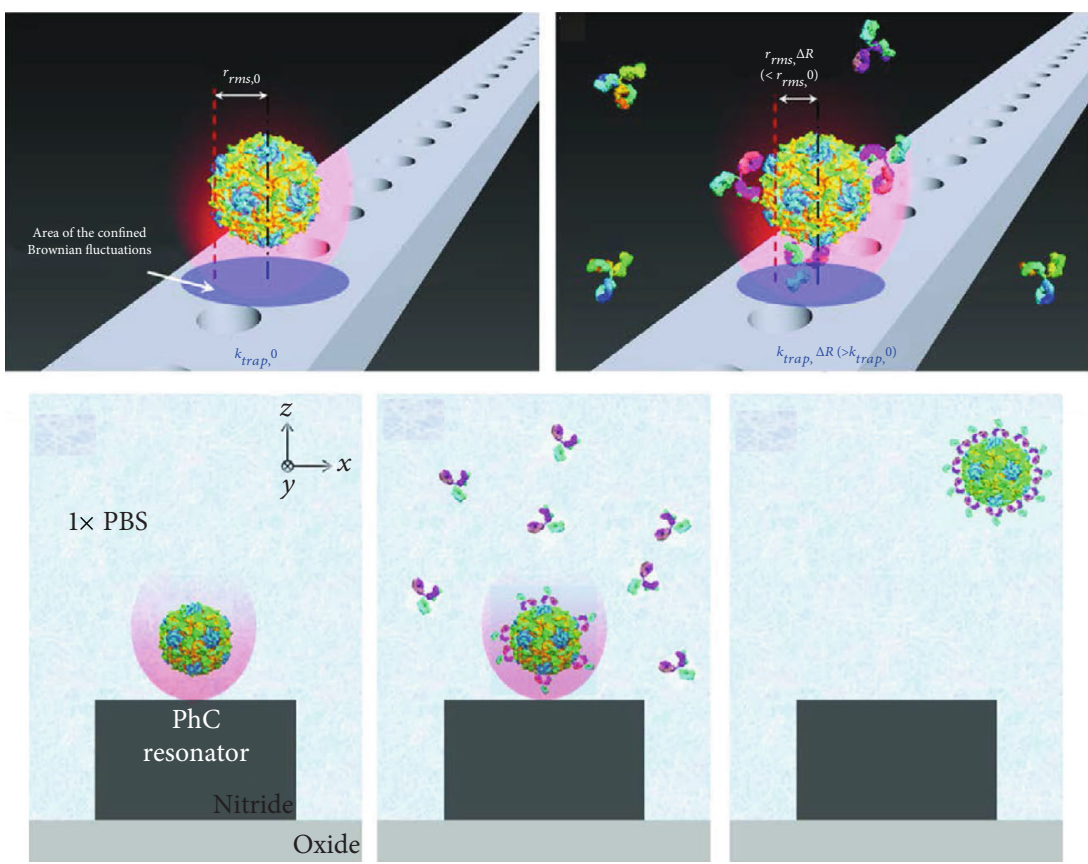

(b)

FIGURE 3: Light-particle interaction for biological applications. (a) The nanowaveguide array for shape-selective sieving of bacteria. S. aureus was trapped in the nanowaveguide array, but E. coli flowed through the array, reprinted with permission from ref. [70]. (b) Rapping of single influenza virus on the photonic crystal nanowaveguide and binding of antibody, reprinted with permission from ref. [23].

static deformation of $20 \mathrm{~nm}$ was demonstrated using a continuous laser power of $3 \mathrm{~mW}$ [43]. Ren et al. studied the nonlinear deformation of a ring resonator with a suspending arc and its pull-back instability (Figure 4(a)) [42]. When the ring arc is deflected by the optical force to an extreme position, a mechanical force pulls the arc abruptly back to its original 

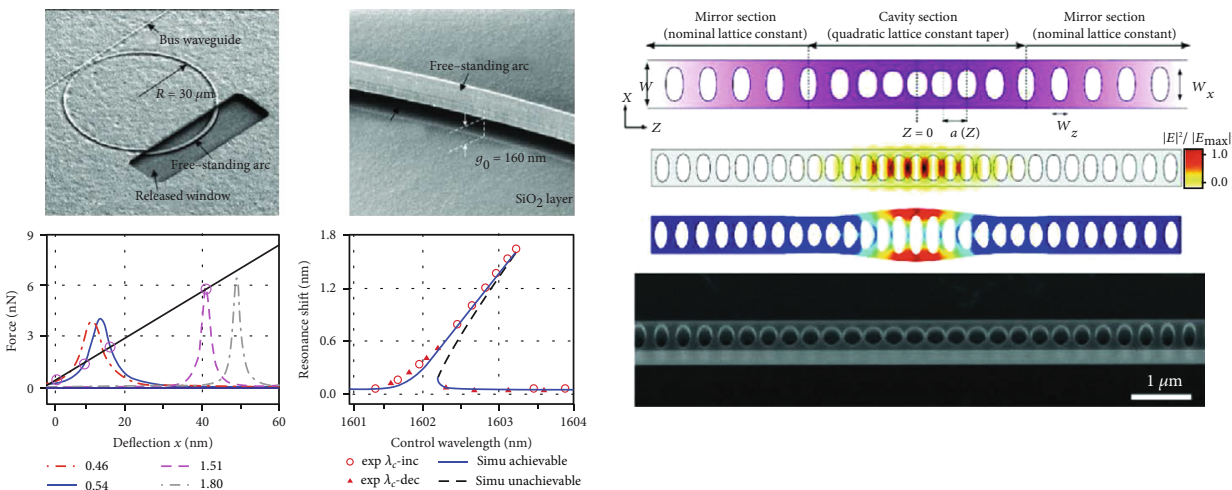

(a)
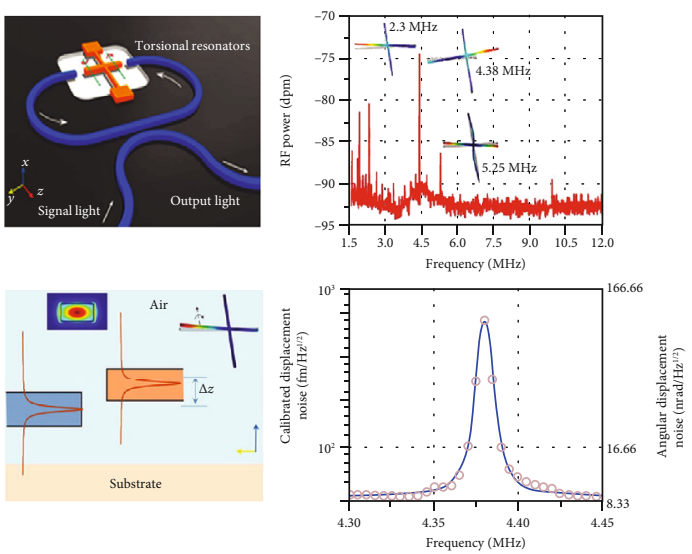

(c)

FIgURE 4: Silicon optomechanical devices. (a) Nonlinear deformation of a ring resonator with a suspended arc with pull-back instability, reprinted with permission from ref. [42]. (b) Optomechanical crystal: geometry, optical resonance, mechanical mode, and SEM image, reprinted with permission from ref. [74]. (c) Torsional frequency mixing and sensing in optomechanical resonators, reprinted with permission from ref. [75].

position as the optical force no longer sustain the mechanical deflection. At the point when the mechanical force becomes dominant, the arc is pulled back. A maximum deformation of $43.1 \mathrm{~nm}(2.8 \mathrm{~mW})$ was demonstrated before pull-back occurred. Mechanical resonators can be excited into selfoscillation through dispersive coupling by the enhanced optical force in the ring resonator using blue-detuned light $[77,80]$. This self-oscillation can also be achieved through periodic modulation or dissipative coupling between two vertically off-set suspending nanowaveguides, extending the working range from blue detuning to red detuning range $[81,82]$. Two distinct self-oscillating mechanical resonators connected by a racetrack optical resonator can directly manipulate phonon transfer using light modulation to compensate the frequency mismatch between the two mechanical resonators [83].

Optomechanical crystals, which both act as photonic crystals in manipulating light and phononic crystals in manipulating mechanical vibrations, greatly enhance the light-matter interactions [84]. The optomechanical crystals consist of a silicon nanobeam with rectangular holes formed by thin crossbars, which strongly couple 200 teraherz photons and 2 gigahertz phonon. Since silicon is opaque below $1 \mu \mathrm{m}$ but silicon nitride is transparent over the visible and near-infrared wavelengths, silicon nitride optomechanical crystals were designed, which support TE optical modes at $980 \mathrm{~nm}$ and couple to $4 \mathrm{GHz}$ mechanical modes (Figure 4(b)) [74, 85].

Optical torque in light-matter interactions is induced by angular momentum of circularly or elliptically polarized light. Optical torque was used to actuate rotational motion in silicon optomechanical device through a birefringent nanowaveguide (Figure 4(c)) [75, 86]. The silicon suspending nanowaveguide was designed to support TE and TM modes through geometric anisotropy. The optical torque on the nanowaveguide can then be controlled by varying the polarization parameters of the light, i.e., the mode amplitude $\left(a_{x}, a_{y}\right)$ and phase difference $(\varphi)$ of the TE and TM modes. The optical torque per unit length of the nanowaveguide can be expressed as

$$
\tau(z)=\eta \frac{\Delta n}{c}\left(2 a_{x} a_{y}\right) \cos (\varphi(z))
$$

where $\eta$ is a coefficient accounts for dipole and electrostrictive forces in dielectric materials, and $\Delta n=n_{x}-n_{y}$ is the difference of effective mode index in $x$ - and $y$-directions. 
Its sign and magnitude are determined by the photon polarization states.

To measure Casimir force between nanostructures, silicon beams with nanoscale $\mathrm{T}$-shaped protrusions were designed and fabricated [87]. The gap between the two beams was controlled by an integrated comb actuator, and the force gradient was detected by an integrated force sensor consisting of a vibrating silicon beam. Nonmonotonic Casimir force with respect to displacement was observed from the Tshaped protrusions on the beams. Understanding Casimir force between nanostructures facilitates the design of complex silicon optomechanical devices.

4.2. Physical Applications of Optomechanical Systems. Strong light-matter interactions in optomechanical systems, which are enhanced by high-quality resonant cavities, enable the development of chip-scale pure photonic circuits for optical signal processing [91, 92] and quantum communication [93]. $\mathrm{Li}$ et al. presented a broadband signal amplifier using a microdisk to induce an optical gradient force onto a cantilevered nanowaveguide [94]. A control laser light is coupled into the microdisk ( $Q$-factor of $\left.5 \times 10^{4}\right)$ to induce resonance and modulated at the mechanical resonance frequency of the cantilevered nanowaveguide $(420 \mathrm{~nm}$ width $\times 220 \mathrm{~nm}$ height $\times 22 \mu \mathrm{m}$ length). With the enhancement of both the optical and mechanical resonances, signal amplification is achieved when a signal light is injected into the cantilevered nanowaveguide and detected at the output. A gain factor of three was achieved, and a broadband of light can be applied except the resonant wavelengths of the optical cavity. Potentially, higher gain factor can be obtained with higher $Q$-factor cavity. The coherent wavelength conversion of photons was demonstrated using an optomechanical crystal resonator, which supports mechanical resonance at $4 \mathrm{GHz}$ and two optical resonances in the $S$ $(1,460 \mathrm{~nm})$ and $C(1,545 \mathrm{~nm})$ bands [95]. A red-detuned pump light couples one of the optical resonant modes to the mechanical resonator, and then is converted back into an optical signal at the resonant wavelength of the other optical resonant mode over a $11.2 \mathrm{THz}$ frequency span. The optomechanical crystal nanobeam $(600 \mathrm{~nm}$ width $\times 220 \mathrm{~nm}$ height $\times 10 \mu \mathrm{m}$ length $)$ has a periodic array of air holes with larger holes on both ends of the nanobeam, forming a Bragg-like reflection and resulting a strong confinement of optical and mechanical resonances at the center of the nanobeam [96]. Optical circulation and photon shuttling were also demonstrated in optomechanical resonators $[97,98]$, providing means to control the transportation of photons in photonic circuits for signal processing and computational operations.

Optomechanical cooling $[99,100]$ refers to the reduced thermal noise of the mechanical vibration of a system through the enhanced interaction between the optical field and the mechanical motion. Cooling the mechanical system to its quantum ground state is critical for high precision measurements and quantum information processing. Optomechanical cooling was experimentally demonstrated in a silicon doubly clamped nanobeam with a mirror coated on its surface, using as a back mirror of a single-ended FabryPérot cavity [101]. By detuning the optical frequency relative to the cavity resonance, drastic cooling down to an effective temperature of $10 \mathrm{~K}$ was observed. A hybrid silicon optomechanical system with a suspended graphene membrane acting as one end of a Fabry-Pérot cavity was also designed to experimentally demonstrate optomechanical cooling (Figure 5(a)) [88]. Graphene is a suitable material to be used because it has high strength and Young's modulus, as well as high thermal conductivity and good optical absorption.

Silicon-based microelectromechanical components such as actuators, interferometers, and tunable lasers, have been widely presented. However, microelectromechanical components are difficult to achieve nanoscale resolution and nano-sized dimensions, suffered from high power consumption, long response time, etc. Therefore, silicon nano-optomechanical components are innovated to overcome these hindrances, paving ways for nanoscale photonic devices. Nano-optomechanical actuator driven by optical gradient force using a nanowaveguide with the photonic crystal cavity was demonstrated [102]. The actuator consists of a suspended nanowaveguide ( $310 \mathrm{~nm}$ width $\times 220 \mathrm{~nm}$ height $\times 50 \mu \mathrm{m}$ length), being coupled to a parallel bus waveguide. When light is coupled into the bus waveguide, an attractive optical gradient force produced between the two waveguides, causing the actuator to move in nanoscale displacement. With the integrated $1 \mathrm{D}$ photonic crystal array along the waveguides, the optical force is enhanced by sixfold. A maximum displacement of $67 \mathrm{~nm}$ was achieved with an optical force of $\sim 1 \mathrm{pN} / \mu \mathrm{m} / \mathrm{mW}$ and a response time of $94.5 \mathrm{~ns}$. Nano-actuator with a mechanical actuation arc controlled by varying the $Q$-factor of the microring resonator was also shown [103]. The Q-factor of the ring resonator was tuned using a $\mathrm{p}$-i-n electro-optics modulator. When current across the $\mathrm{p}-\mathrm{i}-\mathrm{n}$ junction is increased, the $Q$-factor and optical gradient force are reduced. A displacement up to $14 \mathrm{~nm}$ with a resolution of $0.8 \mathrm{~nm}$ was demonstrated. Phase shifters often used in photonic networks such as the Mach Zehnder interferometer to control the output intensity for signal modulation and switching. An optomechanical phase shifter can be designed using a nanowaveguide and a double-clamped suspended beam placed in parallel [104]. Simulation showed that the $180^{\circ}$ phase difference can be obtained, and higher optical power is required for larger gap between the nanowaveguide and the suspended beam or shorter length.

Ren et al. presented a tunable laser with an optomechanical coupled ring reflector [105]. The optomechanical reflector consists of two ring resonators, a driving ring with a suspended arc and a reference ring. The lasing wavelength is selected when it matches with the resonance wavelength of the ring resonators. When the lasing light coupled in the driving ring resonator, the suspended arc is deflected by optical gradient force, changing the resonant wavelength and providing an optical feedback. At a fixed optical power, a balance between the optical force and mechanical force in the arc leads to a stable lasing wavelength. The silicon optomechanical tunable laser has a tuning range of $13.3 \mathrm{~nm}$ with a tuning coefficient of $127 \mathrm{GHz} / \mathrm{nm}$. The ring resonator was also used to enhance the optical gradient force acted on a double-clamped silicon nanowire, inducing bistability that represents the two memory states 0 and 1 (Figure 5(b)) [89]. Both states can be easily set and reset by modulating 


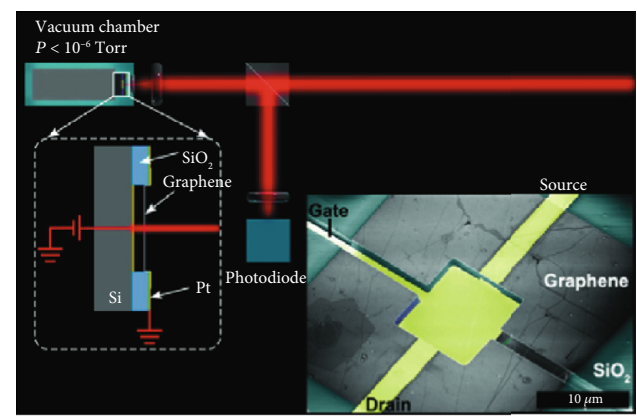

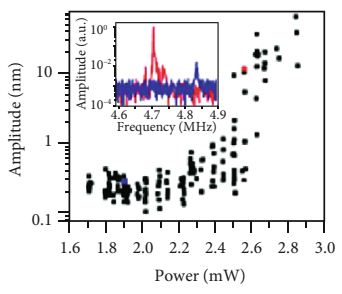

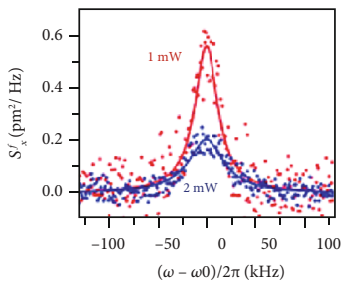

(a)
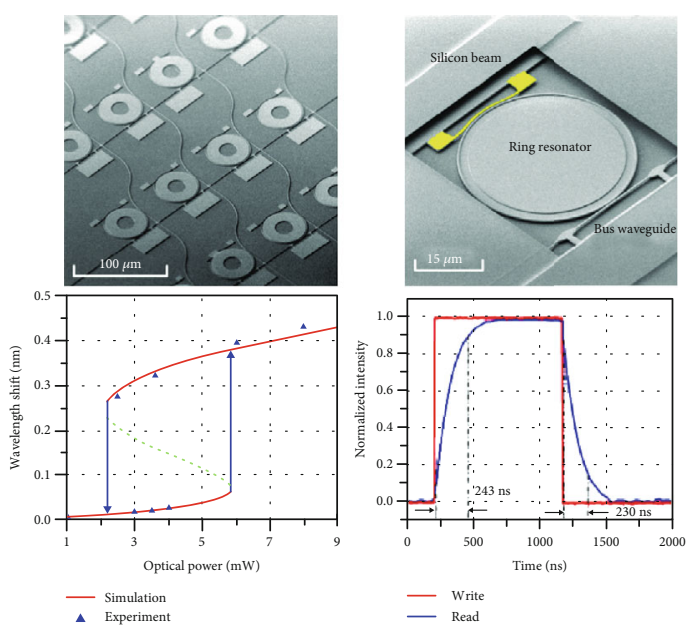

二 Write $_{\text {Read }}$

(b)
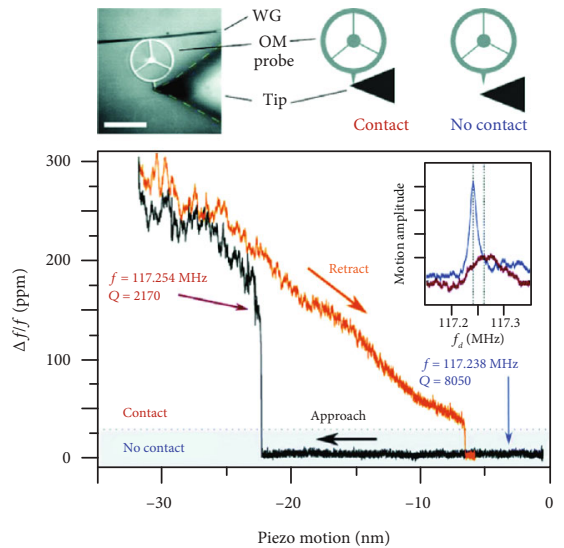

(c)

FIgURE 5: Applications of silicon optomechanical devices. (a) Photothermal self-oscillation and laser cooling of graphene optomechanical systems, reprinted with permission from ref. [88]. (b) Silicon nano-wire memory driven by gradient optical force enhanced by the ring resonator. Two stable deformation positions represent two memory states (0 and 1), reprinted with permission from ref. [89]. (c) Resonating optomechanical atomic force probe operated at a frequency of $117 \mathrm{MHz}$, two decades above cantilevers, with a Brownian motion amplitude four orders below, reprinted with permission from ref. [90].

the input light that is less than $3 \mathrm{~mW}$ with a response time $<250 \mathrm{~ns}$.

Silicon optomechanical systems are also developed for sensing applications [106-108]. The photonic crystal splitbeam nanocavity made of two cantilever resonators was optimized to detect nano-scale torque based on dissipative and dispersive optomechanical coupling [109]. When the gap of the split-beam nanocavity is mechanically modified, the nanocavity length is effectively changed, leading to a dispersive coupling to the optical frequency. Consequently, the nanocavity photon decay rate is also strongly depending on the gap, leading to dissipative optomechanical coupling [110]. Both couplings enable subpg torque sensing with a sensitivity of $1.2 \times 10^{-20} \mathrm{Nm}\left(\mathrm{Hz}^{-0.5}\right)$ in ambient conditions, allowing sensitive readout in nanomagnetic and mesoscopic systems. The hybrid optomechanical torque sensor by integrating the mesoscopic ferromagnetic needle onto an arced torsional resonator was demonstrated for the studies of nanomagnetism [111]. A torque is induced by applying an external magnetic field perpendicular to the magnetic moment of the needle. By designing a feedback loop using the measured mechanical signal, the resonator motion can be amplified to detect mechanical motion in damped environment or dampened to enable faster measurements without sacrificing sensitivity.

Optomechanical mass sensors normally use the change of mechanical oscillating frequency of the optical resonator to detect the mass of deposited molecules $\left(\Delta m \propto \Delta \omega_{n}\right)$. A nonlinear optical mass sensor was proposed using a toroidal nanocavity to measure the mass of molecules such as human chromosomes-1 [112]. For mass measurement, a strong pump light and a weak signal light are applied to detect the cavity vibrational frequency $\left(\omega_{n}\right)$. The pump light frequency matches with the cavity resonant frequency, and the weak 
signal is applied to detect the nonlinear transmission spectrum. When the beat frequency between the two lights approaching $\omega_{n}$, nonlinear Stokes scattering occurs, and the transmission peak with respect to signal-cavity detuning occurs at $-\omega_{n}$. The nonlinear mass sensing approach is superior to linear equivalent because it is less affected by detection noise. Recently, the atomic force sensor using optomechanical resonating probe was also demonstrated (Figure 5(c)) [90]. A ring resonator with a protruding apex was designed, having an optical resonant wavelength of $1,552 \mathrm{~nm}$ and optical Q-factor of $7 \times 10^{4}$ and mechanical resonant frequency of $\sim 117 \mathrm{MHz}$ and mechanical $Q$-factor of $10^{3}$. The force gradient approaching a contact was measured by monitoring the probe mechanical frequency variations. The fully optically actuated and detected atomic force sensing probe achieves a frequency 2 decades higher than commercially instruments and a 4 order lower Brownian motion.

\section{Discussion and Future Perspective}

In this review, light-matter interactions from particles and nanostructures in silicon nanophotonic and optomechanical devices are discussed. Silicon nanofabrication technology has advanced and revolutionized near-field optical manipulation of nanoparticles and nanostructures with highly localized light field by realizing versatile nanoscale structures with high precision and repeatability. Silicon is not only a great material for optomechanical system but it is also compatible for hybrid device integration with other elegant materials. Diamond thin film can be deposited on silicon dioxide substrate to realize an efficient optomechanical transduction via optical gradient forces [113]. Its high Young's modulus, superior thermal properties, and wide electronic bandgap with broadband transparency make it suitable for high-quality nanophotonic devices. Zhang et al. used a phase change material of $\mathrm{Ge}_{2} \mathrm{Sb}_{2} \mathrm{Te}_{5}$ (GST) to fabricate a nanowaveguide $(800 \mathrm{~nm}$ width and $500 \mathrm{~nm}$ height) on a silicon oxide wafer [47]. The GST material can be switched between amorphous and crystalline states through laser pulse heating. By switching between these two states, they demonstrated that a continuous optical pushing or pulling force with the same order of magnitude acted on $50 \mathrm{~nm}$ gold particles. Lin et al. also demonstrated a hybrid plasmonic nano-taper coupled on top of a silicon nitride waveguide [114]. In this design, the waveguide mode excites two hybrid plasmonic modes at the base of the nano-taper, transferring the optical energy from the waveguide to the nano-taper. Subsequently, the optical energy is condensed at the tip of the nano-taper, generating a strong optical force for nanoparticle trapping. Stable trapping of single $100 \mathrm{~nm}$ polystyrene particle was achieved with a low threshold input power of $3.57 \mathrm{~mW}$.

Many efforts from single nanowaveguide and waveguide pairs to photonic crystals have been devoted to exploring the trapping limit in size and efficiency. With over onedecade development, the trapping size reaches $60 \mathrm{~nm}$ polystyrene nanoparticles [40], DNA [20] and viruses [23], and the trapping efficiency is near to $100 \%$ [70]. Hundreds of bacteria and nanoparticles can also simultaneously be han- dled in a single chip [70, 71]. Further development in the design of a large scale and subwavelength hotspots on a silicon device could also advance in the massive trapping and sorting of tiny biomolecules, such as exosome and protein, pushing the size limit to a smaller scale. The design of silicon nanostructures enables a dynamic tuning of hotspot, which could also endow us the ability to control the position and movement of biomolecules precisely and demonstrate a wide range of applications in biomedical sciences.

Recently, other sophisticated phenomena using optical forces emerge, including Casimir force [36], optical nanomotors [13], particle rotation by spin and orbital angular momentum of light [115], and metasurface-enhanced optical force [116]. These explorations show intriguing features that could be used to facilitate the optical trapping and sorting. Newly emerged optical phenomena such as bound state in the continuum, spin-orbit interactions can also be utilized to empower versatile manipulations of biomolecules such as viruses and exosome $[117,118]$ and synthesized particles with different chiralities and shapes [119]. Some other forces could also involve in the versatile manipulations with the optical forces, including photopheric force [120], fluidic drag force [15], and Brownian force [121].

With the synergy of microfluidic technology, rapid manipulation of $50 \mathrm{~nm}$ gold nanoparticles in a high-speed flow stream (e.g., $450 \mu \mathrm{m} / \mathrm{s}$ ) was explored [122], showing the future potentials of optical force-based silicon nanophotonics in handling biological particles for high-throughput applications. Moreover, on-chip-integrated LaguerreGaussian and Bessel beams $[123,124]$ or the interaction of light with nonuniform fluids to realize tunable and reconfigurable optical forces [125] offer new approaches in bioparticle manipulation. Recently, Hu et al. integrated acoustic force with optical force in a single optofluidic chip to achieve a precise and specific leukocyte separation, which uses acoustic force to separate granulocytes based on size and optical force to separate lymphocytes and monocytes based on refractive index differences, showing a greater potential of hybrid systems in biomedical applications [126].

The optical forces in optomechanics, on the other hand, help cool nanostructures towards quantum regime in the room temperature [93] and also benefit various utilities in the signal storage and processing [28], photon and phonon manipulations [83], optical sensing [127], etc. Meanwhile, more demonstrations on the photon and phonon manipulations could also be done using the intriguing physics in optomechanics such as superfluid [128], minimizing the quantum devices for faster computing and information processing. Last but not least, we could also expect more intriguing effect using the optical forces in the silicon chip. For example, optical force triggered nanorobots for drug targeting and energy conversion at nanoscale.

\section{Conflicts of Interest}

The authors declare that there is no conflict of interest regarding the publication of this article. 


\section{Authors' Contributions}

L. K. Chin, Y. Z. Shi, and A. Q. Liu create the outline of the manuscript. L. K. Chin and Y. Z. Shi wrote the manuscript. A. Q. Liu revised the manuscript. Lip Ket Chin and Yuzhi Shi contributed equally to this work.

\section{Funding}

This work was supported by the Singapore National Research Foundation [NRFCRP13-2014-01].

\section{References}

[1] P. D. Lett, R. N. Watts, C. I. Westbrook, W. D. Phillips, P. L. Gould, and H. J. Metcalf, "Observation of atoms laser cooled below the Doppler limit," Physical Review Letters, vol. 61, no. 2, pp. 169-172, 1988.

[2] C. N. Cohen-Tannoudji and W. D. Phillips, "New mechanisms for laser cooling," Physics Today, vol. 43, no. 10, pp. 33-40, 1990.

[3] A. Ashkin, J. M. Dziedzic, J. E. Bjorkholm, and S. Chu, "Observation of a single-beam gradient force optical trap for dielectric particles,” Optics Letters, vol. 11, no. 5, p. 288, 1986.

[4] A. Ashkin and J. M. Dziedzic, "Optical trapping and manipulation of viruses and bacteria," Science, vol. 235, no. 4795, pp. 1517-1520, 1987.

[5] D. G. Grier, "A revolution in optical manipulation," Nature, vol. 424, no. 6950, pp. 810-816, 2003.

[6] L. K. Chin, A. Q. Liu, C. S. Lim et al., "Differential single living cell refractometry using grating resonant cavity with optical trap," Applied Physics Letters, vol. 91, no. 24, article 243901, 2007.

[7] X. Hu, D. Zhu, M. Chen et al., "Precise and non-invasive circulating tumor cell isolation based on optical force using homologous erythrocyte binding," Lab on a Chip, vol. 19, no. 15, pp. 2549-2556, 2019.

[8] M. L. Juan, M. Righini, and R. Quidant, "Plasmon nanooptical tweezers," Nature Photonics, vol. 5, no. 6, pp. 349356, 2011.

[9] M. Liu, T. Zentgraf, Y. Liu, G. Bartal, and X. Zhang, "Lightdriven nanoscale plasmonic motors," Nature Nanotechnology, vol. 5, no. 8, pp. 570-573, 2010.

[10] H. Cai, B. Liu, X. M. Zhang et al., "A micromachined tunable coupled-cavity laser for wide tuning range and high spectral purity," Optics Express, vol. 16, no. 21, pp. 16670-16679, 2008.

[11] A. Q. Liu and X. M. Zhang, "A review of MEMS externalcavity tunable lasers," Journal of Micromechanics and Microengineering, vol. 17, no. 1, pp. R1-R13, 2007.

[12] Y. M. Sabry, D. Khalil, and T. Bourouina, "Monolithic silicon-micromachined free-space optical interferometers onchip," Laser \& Photonics Reviews, vol. 9, no. 1, pp. 1-24, 2015.

[13] Y. Shi, T. Zhu, K. T. Nguyen et al., "Optofluidic microengine in a dynamic flow environment via self-induced backaction," ACS Photonics, vol. 7, no. 6, pp. 1500-1507, 2020.

[14] M. L. Juan, R. Gordon, Y. Pang, F. Eftekhari, and R. Quidant, "Self-induced back-action optical trapping of dielectric nanoparticles," Nature Physics, vol. 5, no. 12, pp. 915-919, 2009.

[15] Y. Shi, S. Xiong, L. K. Chin et al., "Nanometer-precision linear sorting with synchronized optofluidic dual barriers," Science Advances, vol. 4, no. 1, article eaao0773, 2018.

[16] C. Pin, R. Otsuka, and K. Sasaki, "Optical transport and sorting of fluorescent nanodiamonds inside a tapered glass capillary: optical sorting of nanomaterials at the Femtonewton scale," ACS Applied Nano Materials, vol. 3, no. 5, pp. 4127-4134, 2020.

[17] J. E. Melzer and E. McLeod, "Fundamental limits of optical tweezer nanoparticle manipulation speeds," ACS Nano, vol. 12, no. 3, pp. 2440-2447, 2018.

[18] S. Lin, E. Schonbrun, and K. Crozier, "Optical manipulation with planar silicon microring resonators," Nano Letters, vol. 10, no. 7, pp. 2408-2411, 2010.

[19] N. Gaber, M. Malak, F. Marty, D. E. Angelescu, E. Richalot, and T. Bourouina, "Optical trapping and binding of particles in an optofluidic stable Fabry-Pérot resonator with singlesided injection," Lab on a Chip, vol. 14, no. 13, pp. 22592265, 2014.

[20] A. H. J. Yang, S. D. Moore, B. S. Schmidt, M. Klug, M. Lipson, and D. Erickson, "Optical manipulation of nanoparticles and biomolecules in sub-wavelength slot waveguides," Nature, vol. 457, no. 7225, pp. 71-75, 2009.

[21] L. C. Botten, T. P. White, A. A. Asatryan, T. N. Langtry, C. M. de Sterke, and R. C. McPhedran, "Bloch mode scattering matrix methods for modeling extended photonic crystal structures. I. Theory," Physical Review E, vol. 70, no. 5, article 056606, 2004.

[22] M. Tardif, J.-B. Jager, P. R. Marcoux et al., "Single-cell bacterium identification with a SOI optical microcavity," Applied Physics Letters, vol. 109, no. 13, article 133510, 2016.

[23] P. Kang, P. Schein, X. Serey, D. O’Dell, and D. Erickson, "Nanophotonic detection of freely interacting molecules on a single influenza virus," Scientific Reports, vol. 5, no. 1, article 12087, 2015.

[24] S. B. Wang and C. T. Chan, "Lateral optical force on chiral particles near a surface," Nature Communications, vol. 5, no. 1, article 3307, 2014.

[25] F. J. Rodríguez-Fortuño, N. Engheta, A. Martínez, and A. V. Zayats, "Lateral forces on circularly polarizable particles near a surface," Nature Communications, vol. 6, no. 1, article 8799, 2015.

[26] X. Cai, J. Wang, M. J. Strain et al., "Integrated compact optical vortex beam emitters," Science, vol. 338, no. 6105, pp. 363366, 2012.

[27] M. Padgett and R. Bowman, "Tweezers with a twist," Nature Photonics, vol. 5, no. 6, pp. 343-348, 2011.

[28] M. Li, W. H. P. Pernice, C. Xiong, T. Baehr-Jones, M. Hochberg, and H. X. Tang, "Harnessing optical forces in integrated photonic circuits," Nature, vol. 456, no. 7221, pp. 480-484, 2008.

[29] W. H. P. Pernice, M. Li, K. Y. Fong, and H. X. Tang, "Modeling of the optical force between propagating lightwaves in parallel 3D waveguides," Optics Express, vol. 17, no. 18, pp. 16032-16037, 2009.

[30] W. H. P. Pernice, M. Li, and H. X. Tang, "Theoretical investigation of the transverse optical force between a silicon nanowire waveguide and a substrate," Optics Express, vol. 17, no. 3, pp. 1806-1816, 2009. 
[31] G. S. Wiederhecker, S. Manipatruni, S. Lee, and M. Lipson, "Broadband tuning of optomechanical cavities," Optics Express, vol. 19, no. 3, pp. 2782-2790, 2011.

[32] L. Midolo, A. Schliesser, and A. Fiore, "Nano-opto-electromechanical systems," Nature Nanotechnology, vol. 13, no. 1, pp. 11-18, 2018.

[33] D. Van Thourhout and J. Roels, "Optomechanical device actuation through the optical gradient force," Nature Photonics, vol. 4, no. 4, pp. 211-217, 2010.

[34] V. Kajorndejnukul, W. Ding, S. Sukhov, C.-W. Qiu, and A. Dogariu, "Linear momentum increase and negative optical forces at dielectric interface," Nature Photonics, vol. 7, no. 10, pp. 787-790, 2013.

[35] M. Boström and B. E. Sernelius, "Thermal effects on the casimir force in the $0.1-5 \mu \mathrm{m}$ range," Physical Review Letters, vol. 84, no. 20, pp. 4757-4760, 2000.

[36] R. Zhao, L. Li, S. Yang et al., "Stable Casimir equilibria and quantum trapping," Science, vol. 364, no. 6444, pp. 984987, 2019.

[37] A. W. Rodriguez, F. Capasso, and S. G. Johnson, "The Casimir effect in microstructured geometries," Nature Photonics, vol. 5, no. 4, pp. 211-221, 2011.

[38] W. H. P. Pernice, M. Li, D. Garcia-Sanchez, and H. X. Tang, "Analysis of short range forces in opto-mechanical devices with a nanogap," Optics Express, vol. 18, no. 12, pp. 1261512621, 2010.

[39] Y. Shi, H. Zhao, L. K. Chin et al., "Optical potential-well array for high-selectivity, massive trapping and sorting at nanoscale," Nano Letters, vol. 20, no. 7, pp. 5193-5200, 2020.

[40] S. Mandal, X. Serey, and D. Erickson, "Nanomanipulation using silicon photonic crystal resonators," Nano Letters, vol. 10, no. 1, pp. 99-104, 2010.

[41] E. Jaquay, L. J. Martínez, C. A. Mejia, and M. L. Povinelli, "Light-assisted, templated self-assembly using a photoniccrystal slab," Nano Letters, vol. 13, no. 5, pp. 2290-2294, 2013.

[42] M. Ren, J. Huang, H. Cai et al., "Nano-optomechanical actuator and pull-back instability," ACS Nano, vol. 7, no. 2, pp. 1676-1681, 2013.

[43] G. S. Wiederhecker, L. Chen, A. Gondarenko, and M. Lipson, "Controlling photonic structures using optical forces," Nature, vol. 462, no. 7273, pp. 633-636, 2009.

[44] C. Pin, J. B. Jager, M. Tardif et al., "Optical tweezing using tunable optical lattices along a few-mode silicon waveguide," Lab on a Chip, vol. 18, no. 12, pp. 1750-1757, 2018.

[45] S. Gaugiran, S. Gétin, J. M. Fedeli et al., "Optical manipulation of microparticles and cells on silicon nitride waveguides," Optics Express, vol. 13, no. 18, pp. 6956-6963, 2005.

[46] H. Cai and A. W. Poon, "Optical trapping of microparticles using silicon nitride waveguide junctions and taperedwaveguide junctions on an optofluidic chip," Lab on a Chip, vol. 12, no. 19, pp. 3803-3809, 2012.

[47] T. Zhang, S. Mei, Q. Wang, H. Liu, C. T. Lim, and J. Teng, "Reconfigurable optical manipulation by phase change material waveguides," Nanoscale, vol. 9, no. 20, pp. 6895-6900, 2017.

[48] O. G. Hellesø, P. Løvhaugen, A. Z. Subramanian, J. S. Wilkinson, and B. S. Ahluwalia, "Surface transport and stable trapping of particles and cells by an optical waveguide loop," Lab on a Chip, vol. 12, no. 18, pp. 3436-3440, 2012.
[49] A. H. J. Yang, T. Lerdsuchatawanich, and D. Erickson, "Forces and transport velocities for a particle in a slot waveguide," Nano Letters, vol. 9, no. 3, pp. 1182-1188, 2009.

[50] S. Zhang, Z. Yong, Y. Shi, and S. He, "Numerical analysis of an optical nanoscale particles trapping device based on a slotted nanobeam cavity," Scientific Reports, vol. 6, no. 1, article 35977, 2016.

[51] S. Lin and K. B. Crozier, "An integrated microparticle sorting system based on near-field optical forces and a structural perturbation," Optics Express, vol. 20, no. 4, pp. 3367-3374, 2012

[52] X. Xu, G. Wang, W. Jiao, W. Ji, M. Jiang, and X. Zhang, "Multi-level sorting of nanoparticles on multi-step optical waveguide splitter," Optics Express, vol. 26, no. 22, pp. 29262-29271, 2018.

[53] V. W. L. Ho, Y. Chang, Y. Liu et al., "Optical trapping and manipulating with a silica microring resonator in a selflocked scheme," Micromachines, vol. 11, no. 2, p. 202, 2020.

[54] J. Witzens and M. Hochberg, "Optical detection of target molecule induced aggregation of nanoparticles by means of high-Q resonators," Optics Express, vol. 19, no. 8, pp. 70347061, 2011.

[55] A. H. J. Yang and D. Erickson, "Optofluidic ring resonator switch for optical particle transport," Lab on a Chip, vol. 10, no. 6, pp. 769-774, 2010.

[56] W. Xu, Y. Wang, W. Jiao et al., "Tunable optofluidic sorting and manipulation on micro-ring resonators from a statistics perspective," Optics Letters, vol. 44, no. 13, pp. 3226-3229, 2019.

[57] X. Xu, Y. Dong, G. Wang et al., "Reconfigurable sorting of nanoparticles on a thermal tuning silicon based optofluidic chip," IEEE Photonics Journal, vol. 10, no. 1, pp. 1-7, 2018.

[58] A. Rahmani and P. C. Chaumet, "Optical trapping near a photonic crystal," Optics Express, vol. 14, no. 13, pp. 63536358, 2006.

[59] P. T. Lin, T. W. Lu, and P. T. Lee, "Photonic crystal waveguide cavity with waist design for efficient trapping and detection of nanoparticles," Optics Express, vol. 22, no. 6, pp. 6791-6800, 2014.

[60] D. Yang, F. Gao, Q.-T. Cao, C. Wang, Y. Ji, and Y.-F. Xiao, "Single nanoparticle trapping based on on-chip nanoslotted nanobeam cavities," Photonics Research, vol. 6, no. 2, p. 99, 2018.

[61] Y. Gao and Y. Shi, "Design of a single nanoparticle trapping device based on bow-tie-shaped photonic crystal nanobeam cavities," IEEE Photonics Journal, vol. 11, no. 3, pp. 1-8, 2019.

[62] P. Jing, J. Wu, and L. Y. Lin, "Patterned optical trapping with two-dimensional photonic crystals," ACS Photonics, vol. 1, no. 5, pp. 398-402, 2014.

[63] E. Jaquay, L. J. Martínez, N. Huang, C. A. Mejia, D. Sarkar, and M. L. Povinelli, "Light-assisted, templated self-assembly of gold nanoparticle chains," Nano Letters, vol. 14, no. 9, pp. 5184-5188, 2014.

[64] H. Xin, Y. Li, Y. C. Liu, Y. Zhang, Y. F. Xiao, and B. Li, "Optical forces: from fundamental to biological applications," Advanced Materials, vol. 32, no. 37, article e2001994, 2020.

[65] A. Ahmad, V. Dubey, V. R. Singh et al., "Quantitative phase microscopy of red blood cells during planar trapping and propulsion," Lab on a Chip, vol. 18, no. 19, pp. 3025-3036, 2018 . 
[66] Y. Li, X. Liu, and B. Li, "Single-cell biomagnifier for optical nanoscopes and nanotweezers," Light: Science \& Applications, vol. 8, no. 1, p. 61, 2019.

[67] T. van Leest and J. Caro, "Cavity-enhanced optical trapping of bacteria using a silicon photonic crystal," Lab on a Chip, vol. 13, no. 22, pp. 4358-4365, 2013.

[68] R. Therisod, M. Tardif, P. R. Marcoux et al., "Gram-type differentiation of bacteria with 2D hollow photonic crystal cavities," Applied Physics Letters, vol. 113, no. 11, article 111101, 2018.

[69] D. Conteduca, G. Brunetti, F. Dell'Olio, M. N. Armenise, T. F. Krauss, and C. Ciminelli, "Monitoring of individual bacteria using electro-photonic traps," Biomedical Optics Express, vol. 10, no. 7, pp. 3463-3471, 2019.

[70] Y. Shi, H. Zhao, K. T. Nguyen et al., "Nanophotonic arrayinduced dynamic behavior for label-free shape-selective bacteria sieving," ACS Nano, vol. 13, no. 10, pp. 12070-12080, 2019.

[71] H. Zhao, L. K. Chin, Y. Shi et al., "Massive nanophotonic trapping and alignment of rod-shaped bacteria for parallel single-cell studies," Sensors and Actuators B: Chemical, vol. 306, article 127562, 2020.

[72] M. Soltani, J. Lin, R. A. Forties et al., "Nanophotonic trapping for precise manipulation of biomolecular arrays," Nature Nanotechnology, vol. 9, no. 6, pp. 448-452, 2014.

[73] J. J. Otterstrom, B. Brandenburg, M. H. Koldijk et al., "Relating influenza virus membrane fusion kinetics to stoichiometry of neutralizing antibodies at the single-particle level," Proceedings of the National Academy of Sciences of the United States of America, vol. 111, no. 48, pp. E5143-E5148, 2014.

[74] M. Davanço, S. Ates, Y. Liu, and K. Srinivasan, "Si3N4 optomechanical crystals in the resolved-sideband regime," Applied Physics Letters, vol. 104, no. 4, article 041101, 2014.

[75] J. G. Huang, H. Cai, Y. D. Gu et al., "Torsional frequency mixing and sensing in optomechanical resonators," Applied Physics Letters, vol. 111, no. 11, article 111102, 2017.

[76] M. Li, W. H. P. Pernice, and H. X. Tang, "Tunable bipolar optical interactions between guided lightwaves," Nature Photonics, vol. 3, no. 8, pp. 464-468, 2009.

[77] J. Roels, I. De Vlaminck, L. Lagae, B. Maes, D. Van Thourhout, and R. Baets, "Tunable optical forces between nanophotonic waveguides," Nature Nanotechnology, vol. 4, no. 8, pp. 510-513, 2009.

[78] J. R. Rodrigues and V. R. Almeida, "Tailoring optical forces behavior in nano-optomechanical devices immersed in fluid media," Scientific Reports, vol. 7, no. 1, article 14325, 2017.

[79] P. T. Rakich, M. A. Popović, M. Soljačić, and E. P. Ippen, "Trapping, corralling and spectral bonding of optical resonances through optically induced potentials," Nature Photonics, vol. 1, no. 11, pp. 658-665, 2007.

[80] M. Hossein-Zadeh and K. J. Vahala, "An optomechanical oscillator on a silicon chip," IEEE Journal of Selected Topics in Quantum Electronics, vol. 16, no. 1, pp. 276-287, 2010.

[81] J. G. Huang, Y. Li, L. K. Chin et al., "A dissipative selfsustained optomechanical resonator on a silicon chip," Applied Physics Letters, vol. 112, no. 5, article 051104, 2018.

[82] J. Huang, M. Karim, J. Wu, T. Chen, and A. Liu, "Parametric excitation of optomechanical resonators by periodical modulation," Micromachines, vol. 9, no. 4, p. 193, 2018.

[83] J. Huang, L. K. Chin, H. Cai et al., "Dynamic phonon manipulation by optomechanically induced strong coupling between two distinct mechanical resonators," ACS Photonics, vol. 6, no. 8, pp. 1855-1862, 2019.

[84] M. Eichenfield, J. Chan, R. M. Camacho, K. J. Vahala, and O. Painter, "Optomechanical crystals," Nature, vol. 462, no. 7269, pp. 78-82, 2009.

[85] K. E. Grutter, M. Davanco, and K. Srinivasan, " $\mathrm{Si}_{3} \mathrm{~N}_{4}$ Nanobeam optomechanical crystals," IEEE Journal of Selected Topics in Quantum Electronics, vol. 21, no. 4, pp. 61-71, 2015.

[86] L. He, H. Li, and M. Li, "Optomechanical measurement of photon spin angular momentum and optical torque in integrated photonic devices," Science Advances, vol. 2, no. 9, article e1600485, 2016.

[87] L. Tang, M. Wang, C. Y. Ng et al., "Measurement of nonmonotonic Casimir forces between silicon nanostructures," Nature Photonics, vol. 11, no. 2, pp. 97-101, 2017.

[88] R. A. Barton, I. R. Storch, V. P. Adiga et al., "Photothermal self-oscillation and laser cooling of graphene optomechanical systems," Nano Letters, vol. 12, no. 9, pp. 4681-4686, 2012.

[89] B. Dong, H. Cai, L. K. Chin et al., "A silicon-nanowire memory driven by optical gradient force induced bistability," Applied Physics Letters, vol. 107, no. 26, article 261111, 2015.

[90] P. E. Allain, L. Schwab, C. Mismer et al., "Optomechanical resonating probe for very high frequency sensing of atomic forces," Nanoscale, vol. 12, no. 5, pp. 2939-2945, 2020.

[91] K. Fang, M. H. Matheny, X. Luan, and O. Painter, "Optical transduction and routing of microwave phonons in cavityoptomechanical circuits," Nature Photonics, vol. 10, no. 7, pp. 489-496, 2016.

[92] K. C. Balram, M. I. Davanço, J. D. Song, and K. Srinivasan, "Coherent coupling between radiofrequency, optical and acoustic waves in piezo-optomechanical circuits," Nature Photonics, vol. 10, no. 5, pp. 346-352, 2016.

[93] R. Riedinger, A. Wallucks, I. Marinković et al., "Remote quantum entanglement between two micromechanical oscillators," Nature, vol. 556, no. 7702, pp. 473-477, 2018.

[94] H. Li, Y. Chen, J. Noh, S. Tadesse, and M. Li, "Multichannel cavity optomechanics for all-optical amplification of radio frequency signals," Nature Communications, vol. 3, no. 1, article 1091, 2012.

[95] J. T. Hill, A. H. Safavi-Naeini, J. Chan, and O. Painter, "Coherent optical wavelength conversion via cavity optomechanics," Nature Communications, vol. 3, no. 1, article 1196, 2012.

[96] J. Chan, A. H. Safavi-Naeini, J. T. Hill, S. Meenehan, and O. Painter, "Optimized optomechanical crystal cavity with acoustic radiation shield," Applied Physics Letters, vol. 101, no. 8, article $081115,2012$.

[97] F. Ruesink, J. P. Mathew, M. A. Miri, A. Alù, and E. Verhagen, "Optical circulation in a multimode optomechanical resonator," Nature Communications, vol. 9, no. 1, article 1798, 2018.

[98] H. Li and M. Li, "Optomechanical photon shuttling between photonic cavities," Nature Nanotechnology, vol. 9, no. 11, pp. 913-919, 2014.

[99] F. Marquardt, A. A. Clerk, and S. M. Girvin, "Quantum theory of optomechanical cooling," Journal of Modern Optics, vol. 55, no. 19-20, pp. 3329-3338, 2008.

[100] Y.-C. Liu, Y.-W. Hu, C. W. Wong, and Y.-F. Xiao, "Review of cavity optomechanical cooling," Chinese Physics B, vol. 22, no. 11, article $114213,2013$. 
[101] O. Arcizet, P. F. Cohadon, T. Briant, M. Pinard, and A. Heidmann, "Radiation-pressure cooling and optomechanical instability of a micromirror," Nature, vol. 444, no. 7115, pp. 71-74, 2006.

[102] H. Cai, K. J. Xu, A. Q. Liu et al., "Nano-opto-mechanical actuator driven by gradient optical force," Applied Physics Letters, vol. 100, no. 1, article 013108, 2012.

[103] B. Dong, H. Cai, G. I. Ng et al., "A nanoelectromechanical systems actuator driven and controlled by Q-factor attenuation of ring resonator," Applied Physics Letters, vol. 103, no. 18, article 181105, 2013.

[104] Y. Ozer and S. Kocaman, "Stability Formulation for Integrated Opto-mechanic Phase Shifters," Scientific Reports, vol. 8, no. 1, article 1937, 2018.

[105] M. Ren, H. Cai, L. K. Chin et al., “An opto-mechanical coupled-ring reflector driven by optical force for lasing wavelength control," Applied Physics Letters, vol. 108, no. 8, article 081106, 2016.

[106] A. G. Krause, M. Winger, T. D. Blasius, Q. Lin, and O. Painter, "A high-resolution microchip optomechanical accelerometer," Nature Photonics, vol. 6, no. 11, pp. 768772, 2012.

[107] E. Gavartin, P. Verlot, and T. J. Kippenberg, "A hybrid onchip optomechanical transducer for ultrasensitive force measurements," Nature Nanotechnology, vol. 7, no. 8, pp. 509514, 2012.

[108] C. Doolin, P. H. Kim, B. D. Hauer, A. J. R. MacDonald, and J. P. Davis, "Multidimensional optomechanical cantilevers for high-frequency force sensing," New Journal of Physics, vol. 16, no. 3, article 035001, 2014.

[109] M. Wu, A. C. Hryciw, C. Healey et al., "Dissipative and dispersive optomechanics in a nanocavity torque sensor," Physical Review X, vol. 4, no. 2, 2014.

[110] J. Fan, C. Huang, and L. Zhu, "Optomechanical nonlinearity enhanced optical sensors," Optics Express, vol. 23, no. 3, pp. 2973-2981, 2015.

[111] P. H. Kim, B. D. Hauer, T. J. Clark, F. Fani Sani, M. R. Freeman, and J. P. Davis, "Magnetic actuation and feedback cooling of a cavity optomechanical torque sensor," Nature Communications, vol. 8, no. 1, article 1355, 2017.

[112] J.-J. Li and K.-D. Zhu, "Nonlinear optical mass sensor with an optomechanical microresonator," Applied Physics Letters, vol. 101, no. 14, article 141905, 2012.

[113] P. Rath, S. Khasminskaya, C. Nebel, C. Wild, and W. H. P. Pernice, "Diamond-integrated optomechanical circuits," Nature Communications, vol. 4, no. 1, article 1690, 2013.

[114] Y.-C. Lin and P.-T. Lee, "Efficient optical trapping of Nanoparticle via waveguide-coupled hybrid Plasmonic Nanotaper," IEEE Photonics Journal, vol. 11, no. 3, pp. 1-12, 2019.

[115] K. Wang, E. Schonbrun, P. Steinvurzel, and K. B. Crozier, "Trapping and rotating nanoparticles using a plasmonic nano-tweezer with an integrated heat sink," Nature Communications, vol. 2, no. 1, p. 469, 2011.

[116] D. G. Kotsifaki, V. G. Truong, and S. N. Chormaic, "Fanoresonant, asymmetric, metamaterial-assisted tweezers for single nanoparticle trapping," Nano Letters, vol. 20, no. 5, pp. 3388-3395, 2020.

[117] T. Zhu, Y. Shi, W. Ding et al., "Extraordinary multipole modes and ultra-enhanced optical lateral force by chirality," Physical Review Letters, vol. 125, no. 4, 2020.
[118] H. Li, Y. Cao, L.-M. Zhou et al., "Optical pulling forces and their applications," Advances in Optics and Photonics, vol. 12, no. 2, p. 288, 2020.

[119] Y. Shi, T. Zhu, T. Zhang et al., "Chirality-assisted lateral momentum transfer for bidirectional enantioselective separation," Light: Science \& Applications, vol. 9, no. 1, p. 62, 2020.

[120] J. Lu, H. Yang, L. Zhou et al., "Light-induced pulling and pushing by the synergic effect of optical force and photophoretic force," Physical Review Letters, vol. 118, no. 4, article 043601, 2017.

[121] P. Hänggi and F. Marchesoni, "Artificial Brownian motors: controlling transport on the nanoscale," Reviews of Modern Physics, vol. 81, no. 1, pp. 387-442, 2009.

[122] W. Wu, X. Zhu, Y. Zuo et al., "Precise sorting of gold nanoparticles in a flowing system," ACS Photonics, vol. 3, no. 12, pp. 2497-2504, 2016.

[123] T. Wu, T. A. Nieminen, S. Mohanty et al., "A photon-driven micromotor can direct nerve fibre growth," Nature Photonics, vol. 6, no. 1, pp. 62-67, 2012.

[124] Y. Z. Shi, S. Xiong, L. K. Chin et al., "High-resolution and multi-range particle separation by microscopic vibration in an optofluidic chip," Lab on a Chip, vol. 17, no. 14, pp. 2443-2450, 2017.

[125] J. Zhu, X. Zhu, Y. Zuo et al., "Optofluidics: the interaction between light and flowing liquids in integrated devices," Opto-Electronic Advances, vol. 2, no. 11, pp. 1900070119000710, 2019.

[126] X. J. Hu, H. L. Liu, Y. X. Jin et al., "Precise label-free leukocyte subpopulation separation using hybrid acoustic-optical chip," Lab on a Chip, vol. 18, no. 22, pp. 3405-3412, 2018.

[127] M. Toroš and T. S. Monteiro, "Quantum sensing and cooling in three-dimensional levitated cavity optomechanics," Physical Review Research, vol. 2, no. 2, p. 2, 2020.

[128] X. He, G. I. Harris, C. G. Baker et al., "Strong optical coupling through superfluid Brillouin lasing," Nature Physics, vol. 16, no. 4, pp. 417-421, 2020. 